MATHEMATICS OF COMPUTATION

Volume 71, Number 240, Pages 1781-1797

S 0025-5718(01)01382-5

Article electronically published on November 28, 2001

\title{
NUMERICAL CALCULATION \\ OF THE DENSITY OF PRIME NUMBERS WITH A GIVEN LEAST PRIMITIVE ROOT
}

\author{
A. PASZKIEWICZ AND A. SCHINZEL
}

\begin{abstract}
In this paper the densities $D(i)$ of prime numbers $p$ having the least primitive root $g(p)=i$, where $i$ is equal to one of the initial positive integers less than 32 , have been numerically calculated. The computations were carried out under the assumption of the Generalised Riemann Hypothesis. The results of these computations were compared with the results of numerical frequency estimations.
\end{abstract}

\section{An outline of the method of COMputation}

Let $g(p)$ denote the least primitive root modulo $p$ and $D(i)$ the density of prime numbers with the least primitive root equal to $i$, that is

$$
D(i)=\lim _{x \rightarrow \infty} \pi(x)^{-1} \sum_{\substack{p \leq x \\ g(p)=i}} 1 .
$$

In [3] Elliott and Murata gave the formula

$$
D(i)=\sum_{M}(-1)^{|M|-1} A_{M},
$$

where $M$ runs over all the subsets of the set $\{2,3, \ldots, i\}$ containing $i$. Here $A_{M}$ denotes the conjectural density of primes $p$ such that each $a_{i} \in M$ is a primitive root modulo $p$, expressed by the formula in Lemma 11.5, page 140 of Matthews 4]:

$$
A_{M}=\prod_{p}(1-c(p)) \sum_{a \in G\left(a_{1}, \ldots, a_{n}\right)} \omega(a) f(a),
$$

where $M=\left\{a_{1}, \ldots, a_{n}\right\}$ and $G\left(a_{1}, \ldots, a_{n}\right\}$ is the set of squarefree integers of the form $a=\kappa\left(a_{1}^{\varepsilon_{1}}, \ldots, a_{n}^{\varepsilon_{n}}\right) \equiv 1(\bmod 4), \varepsilon_{i}=0,1, \omega(a)=(-1)^{\sum_{i} \varepsilon_{i}}$ and $\kappa(b)$ is the squarefree part of the number $b$

$$
f(b)=\mu(b) \prod_{p \mid b} \frac{c(p)}{1-c(p)} .
$$

Here $c(p)$ is the natural density of the set of primes $\left\{q: q \equiv 1(\bmod p), q \nmid a_{1}, \ldots, a_{n}\right.$, and at least one of the numbers $a_{1}, \ldots, a_{n}$ is a $p$ th power residue modulo $\left.q\right\}$.

Received by the editor November 29, 1999 and, in revised form, December 26, 2000. 2000 Mathematics Subject Classification. Primary 11Y16; Secondary 11A07, 11M26.

Key words and phrases. Prime, generators, primitive roots, extended Riemann hypothesis. 
Formula (2) is based on the assumption that if $a_{1}^{\varepsilon_{i}}, \ldots, a_{n}^{\varepsilon_{n}}=b^{2}$, then $S=\sum_{i} \varepsilon_{i}$ is even. If $S$ is odd, then $A_{M}=0$. There is a factor $1 / 2^{n}$ missing in (1.4) page 114 of Matthews 4, and this error is repeated in the paper of Elliott and Murata.

In [3] Elliott and Murata derived formulas for the density of prime numbers whose least primitive roots are the initial natural numbers 2,3,5,6 and 7 . They are as follows:

$$
\begin{aligned}
D(2)= & \Delta \\
D(3)= & \Delta_{1}-\Delta_{2} \\
D(5)= & \frac{20}{19} \Delta_{1}-\frac{200}{91} \Delta_{2}+\frac{500}{439} \Delta_{3} \\
D(6)= & \Delta_{1}-\frac{282}{91} \Delta_{2}+\frac{1000}{439} \Delta_{3}, \\
D(7)= & \Delta_{1}-\left(4+\frac{9}{91}+\frac{5}{281}\right) \Delta_{2}+\left(6+\frac{183}{439}+\frac{4826}{67585}+\frac{147193}{29669815}\right) \Delta_{3} \\
& -\left(3+\frac{1107}{2131}+\frac{71825}{1290197}+\frac{26503425}{2749409807}\right) \Delta_{4},
\end{aligned}
$$

where the initial $\Delta_{i}(i=1,2,3,4)$ are

$$
\begin{aligned}
& \Delta_{1}=\prod_{p \geq 2}\left(1-\frac{1}{p(p-1)}\right), \text { Artin's constant, } \\
& \Delta_{2}=\prod_{p \geq 2}\left(1-\frac{2}{p(p-1)}+\frac{1}{p^{2}(p-1)}\right), \\
& \Delta_{3}=\prod_{p \geq 2}\left(1-\frac{3}{p(p-1)}+\frac{3}{p^{2}(p-1)}-\frac{1}{p^{3}(p-1)}\right), \\
& \Delta_{4}=\prod_{p \geq 2}\left(1-\frac{4}{p(p-1)}+\frac{6}{p^{2}(p-1)}-\frac{4}{p^{3}(p-1)}+\frac{1}{p^{4}(p-1)}\right) .
\end{aligned}
$$

The calculation of the successive values of $D(i)$ is illustrated with an example for $i=10$. According to $(1)$,

$$
D(10)=\sum_{M}(-1)^{|M|-1} A_{M},
$$

where $M$ runs over all the subsets of the set $\{2,3,5,6,7,10\}$ containing 10 (from the set of all natural numbers $\leq 10$, we remove the powers $1,4,8,9$, which can never be least primitive roots). Observe that if $M$ contains $a, b$ and $a b$, then $A_{M}=0$, because if $a, b$ are primitive roots for $p$, then $a b$ is not. Therefore,

$$
\begin{aligned}
D(10)= & A_{\{10\}}-A_{\{2,10\}}-A_{\{3,10\}}-A_{\{5,10\}}-A_{\{6,10\}} \\
& -A_{\{7,10\}}+A_{\{2,3,10\}}+A_{\{2,6,10\}}+A_{\{2,7,10\}} \\
& +A_{\{3,5,10\}}+A_{\{3,6,10\}}+A_{\{3,7,10\}}+A_{\{5,6,10\}} \\
& +A_{\{5,7,10\}}+A_{\{6,7,10\}}-A_{\{2,3,7,10\}}-A_{\{2,6,7,10\}} \\
& -A_{\{3,5,7,10\}}-A_{\{3,6,7,10\}}-A_{\{5,6,7,10\}} \\
& -A_{\{3,5,6,10\}}+A_{\{3,5,6,7,10\}} .
\end{aligned}
$$

Now, for each of the 22 sets $M$, listed above we calculate $c(p)$, which we denote by $c(p, M)$ in order to avoid ambiguity. So, if the elements $a_{1}, \ldots, a_{n}$, of the set $M$ are 
multiplicatively independent (that is they do not satisfy any relation of the form $a_{1}^{\alpha_{1}} a_{2}^{\alpha_{2}} \cdots a_{n}^{\alpha_{n}}=1$, where $\alpha_{i}(i=1,2, \ldots, n)$ are integers not all equal to 0$)$, then

$$
c(p, M)=\frac{1}{p-1}\left(1-\left(1-\frac{1}{p}\right)^{|M|}\right)
$$

hence,

$$
\prod_{p \geq 2}(1-c(p, M))=\Delta_{|M|} .
$$

The assumption of the multiplicative independence of the elements holds for all the sets listed above except for $\{3,5,6,10\}$ and $\{3,5,6,7,10\}$ (we have $3 \cdot 5^{-1} \cdot 6^{-1} \cdot 10=$ 1). In order to apply Matthews's formula (2) we compute the sum

$$
S(M)=\sum_{\substack{\varepsilon_{1}=0 \\ a=\kappa\left(a_{1}^{1} \cdots a_{n}^{\varepsilon_{n}}\right)}}^{1} \cdots \sum_{\substack{\varepsilon_{n}=0 \\ 1(\bmod 4)}}^{1}(-1)^{\sum_{i} \varepsilon_{i}} f(|a|, M),
$$

where the additional argument of the function $f$ was added in order to avoid ambiguity, e.g.,

$$
\begin{aligned}
& \text { for } M=\{10\}, S(M)=f(1, M)=1 \\
& \text { for } M=\{2,10\}, S(M)=f(1, M)+f(5, M)=1-\frac{c(5, M)}{1-c(5, M)}=1-\frac{9}{91}=\frac{82}{91} .
\end{aligned}
$$

The first summand corresponds to the choice $\varepsilon_{1}=\varepsilon_{2}=0$; the second to the choice $\varepsilon_{1}=\varepsilon_{2}=1$, etc. The calculation of the values of the coefficients $A_{M}$ for the remaining sets $M$ proceeds similarly up to the set $M=\{5,6,7,10\}$ inclusive. For $M=\{5,6,7,10\}$ we have

$$
\begin{aligned}
S(M)= & f(1, M)-f(5, M)+f(21, M)-f(105, M) \\
= & 1+\frac{c(5, M)}{1-c(5, M)}+\frac{c(3, M)}{1-c(3, M)} \cdot \frac{c(7, M)}{1-c(7, M)} \\
& +\frac{c(5, M)}{1-c(5, M)} \cdot \frac{c(3, M)}{1-c(3, M)} \cdot \frac{c(7, M)}{1-c(7, M)} .
\end{aligned}
$$

The first summand corresponds to the choice $\varepsilon_{1}=\varepsilon_{2}=\varepsilon_{3}=\varepsilon_{4}=0$; the second to the choice $\varepsilon_{1}=1, \varepsilon_{2}=\varepsilon_{3}=\varepsilon_{4}=0$; the third to $\varepsilon_{1}=\varepsilon_{2}=\varepsilon_{3}=\varepsilon_{4}=1$; and the fourth to $\varepsilon_{1}=0, \varepsilon_{2}=\varepsilon_{3}=\varepsilon_{4}=1$.

It remains to consider two special sets $M_{1}=\{3,5,6,10\}$ and $M_{2}=\{3,5,6,7,10\}$. For these sets the formula (3) is not valid and is replaced with

$$
\begin{aligned}
c\left(p, M_{1}\right) & =\frac{4}{p(p-1)}-\frac{6}{p^{2}(p-1)}+\frac{3}{p^{3}(p-1)}, \\
S\left(M_{1}\right) & =2 f\left(1, M_{1}\right)-2 f\left(5, M_{1}\right)=2+2 \frac{c\left(5, M_{1}\right)}{1-c\left(5, M_{1}\right)} .
\end{aligned}
$$


The first summand corresponds to the choice $\varepsilon_{1}=\varepsilon_{3}=\varepsilon_{4}=\varepsilon_{2}$; the second to the choice $\varepsilon_{1}=\varepsilon_{3}=\varepsilon_{4} \neq \varepsilon_{2}$ :

$$
\begin{aligned}
c\left(p, M_{2}\right)= & \frac{5}{p(p-1)}-\frac{10}{p^{2}(p-1)}+\frac{9}{p^{3}(p-1)}-\frac{3}{p^{4}(p-1)} \\
S\left(M_{2}\right)= & 2 f\left(1, M_{2}\right)-2 f\left(5, M_{2}\right)+2 f\left(21, M_{2}\right)-2 f\left(105, M_{2}\right) \\
= & 2+2 \frac{c\left(5, M_{2}\right)}{1-c\left(5, M_{2}\right)}+2 \frac{c\left(3, M_{2}\right)}{1-c\left(3, M_{2}\right)} \cdot \frac{c\left(7, M_{2}\right)}{1-c\left(7, M_{2}\right)} \\
& +2 \frac{c\left(3, M_{2}\right)}{1-c\left(3, M_{2}\right)} \cdot \frac{c\left(5, M_{2}\right)}{1-c\left(5, M_{2}\right)} \cdot \frac{c\left(7, M_{2}\right)}{1-c\left(7, M_{2}\right)} .
\end{aligned}
$$

The first summand corresponds to the choice $\varepsilon_{1}=\varepsilon_{3}=\varepsilon_{3}, \varepsilon_{4}=\varepsilon_{1}+\varepsilon_{3}, \varepsilon_{5}=\varepsilon_{3}$; the second to the chioce $\varepsilon_{1}=\varepsilon_{3} \neq \varepsilon_{2}, \varepsilon_{4}=\varepsilon_{1}+\varepsilon_{3}, \varepsilon_{5}=\varepsilon_{3}$; the third to the choice $\varepsilon_{2}=\varepsilon_{3} \neq \varepsilon_{1}, \varepsilon_{4}=\varepsilon_{1}+\varepsilon_{3}, \varepsilon_{5}=\varepsilon_{3}$; the fourth to the choice $\varepsilon_{1}=\varepsilon_{2} \neq \varepsilon_{3}$, $\varepsilon_{4}=\varepsilon_{1}+\varepsilon_{3}, \varepsilon_{5}=\varepsilon_{3}$.

Proceeding in this way, we can calculate densities $D(i)$ for any positive integer $i$. Beyond $i=10$, the derivation of formulas for $D(i)$ ceases to make sense due to their length. However, the use of a computer makes it possible to extend the computations to some extent. In this paper, by designing an algorithm corresponding to the computational process described above, we computed the values of $D(i)$ for all positive integers $i<32$, which are not powers of integers.

\section{Results OF NUMERICAL COMPUTATIONS}

The following numerical investigations were carried out:

- The densities $D(i)$ of prime numbers $p$ having the least primitive root $g(p)$ equal to $i$ were calculated; the results are illustrated in Table 3

- For every prime number $p<4 \cdot 10^{10}$, the value of its least primitive root was determined.

- The computed densities of prime numbers with given least primitive roots were compared with numerical frequency estimates; the respective values are shown side by side in Table 3 .

- The graphs of densities $D(i)$ for initial values of $i$ were prepared and tabulated with step equal to $10^{9}$, the behaviour of the frequencies of prime numbers with a given least primitive root are illustrated in Figures 1024 From the figures it may be concluded that the densities $D(i)$ are very stable. Oscillations have limited amplitude and a tendency to damp out.

- A graph of the average value of the least primitive root of prime numbers not exceeding $4 \cdot 10^{10}$ was prepared (Figure 25) and tabulated with a step equal to $10^{9}$ (Table 1).

- The growth rate of the least primitive root of a prime number was numerically investigated (see Table 2). The value $g(p)$ of the least primitive root of a prime number $p$ is well majorised by a second degree polynomial of the natural $\operatorname{logarithm}$ of $p$, which agrees with the conjecture by E. Bach [1] about the least primitive prime roots modulo a prime number.

All computations were performed with the aid of more than ten desktop IBM PC computers from Pentium 100 to Pentium III 500 during their idle time. The computations lasted approximately one year. The computational procedures (with some small exceptions) were written in a high level language in order to minimise the risk of programming error. Most of the results were verified using popular 
numerical packages, e.g., MAPLE, GP/PARI. The verification required more time than the actual computations. For checking the number of primes generated, we used Mapes's algorithm, which was found to be the most computationally effective within the range of computations performed.

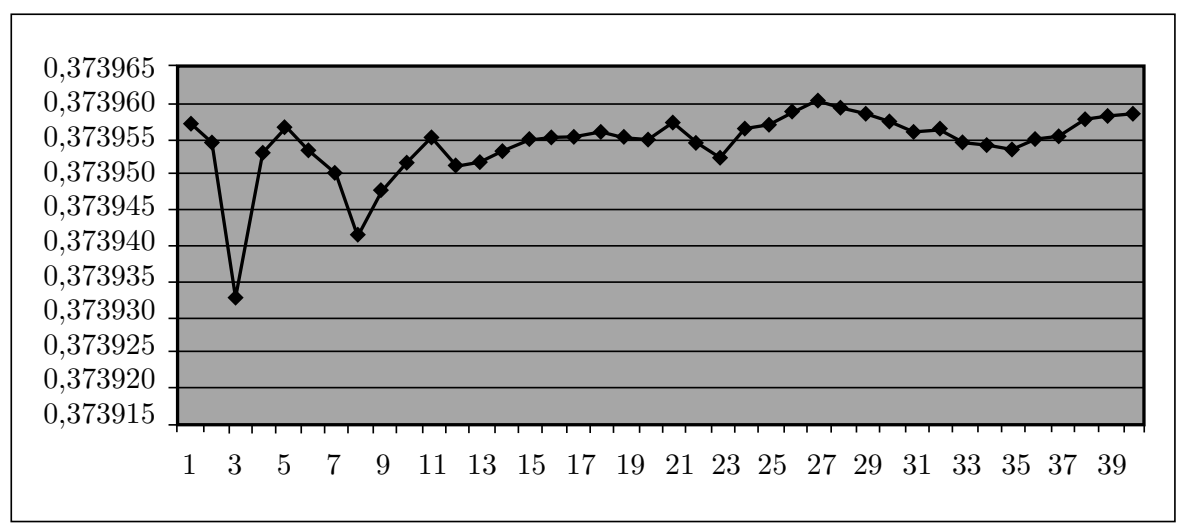

Figure 1 . The density $D(2)$ of prime numbers with the least primitive root equal to 2

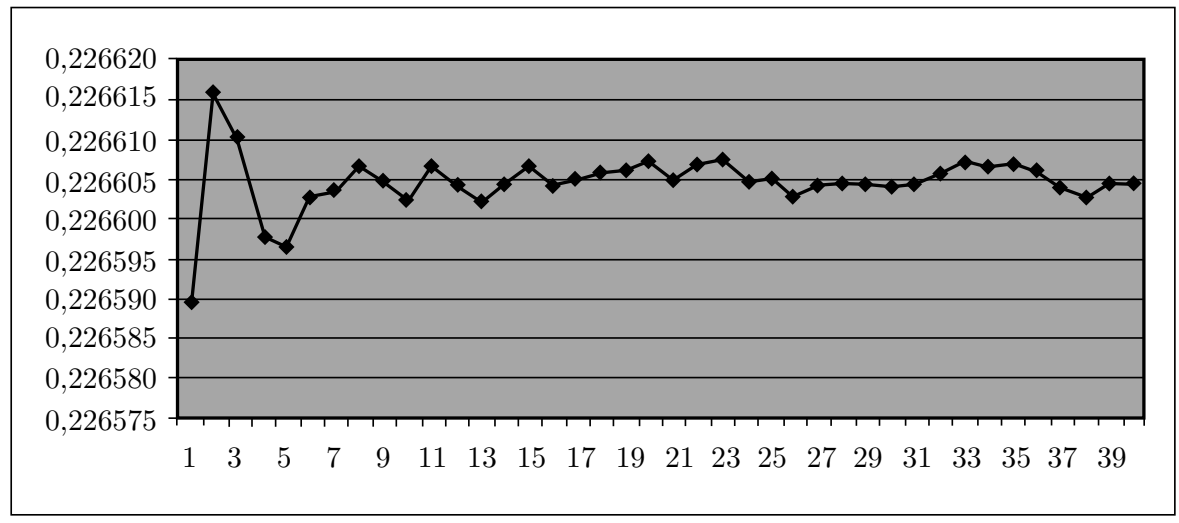

Figure 2 . The density $D(3)$ of prime numbers with the least primitive root equal to 3 


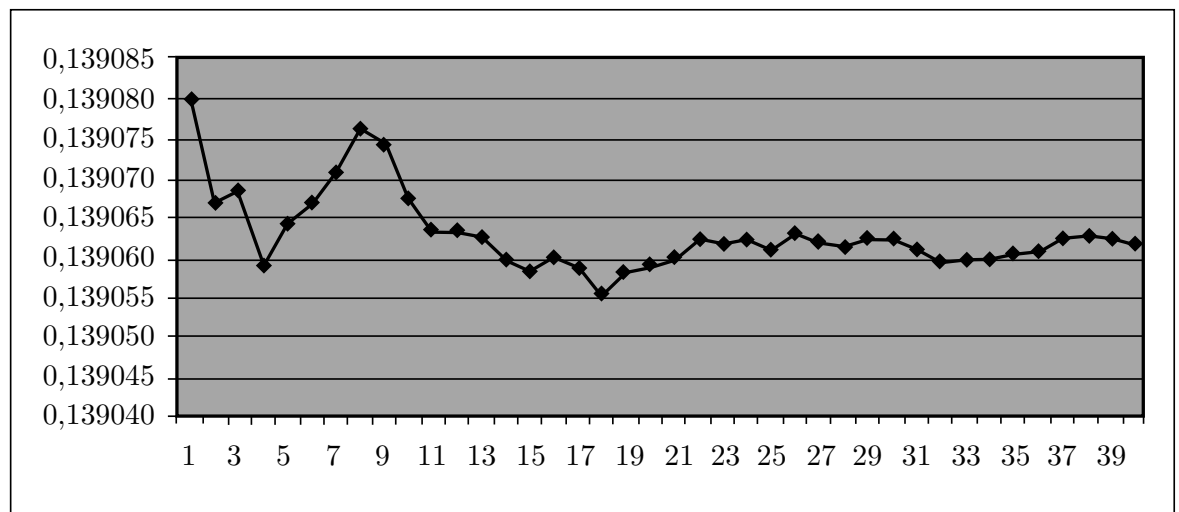

Figure 3 . The density $D(5)$ of prime numbers with the least primitive root equal to 5

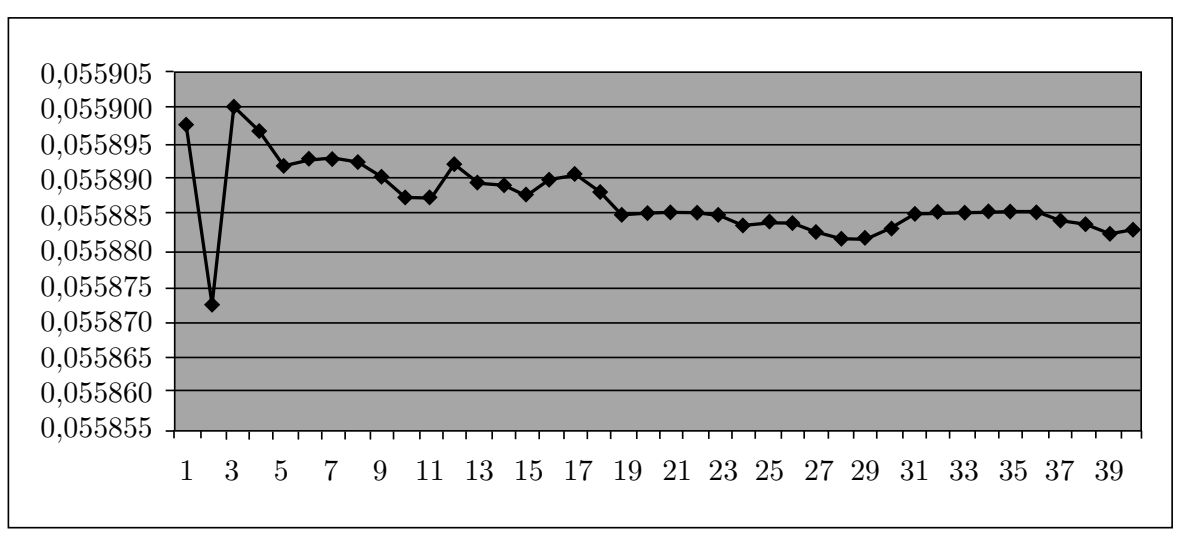

FiguRE 4 . The density $D(6)$ of prime numbers with the least primitive root equal to 6

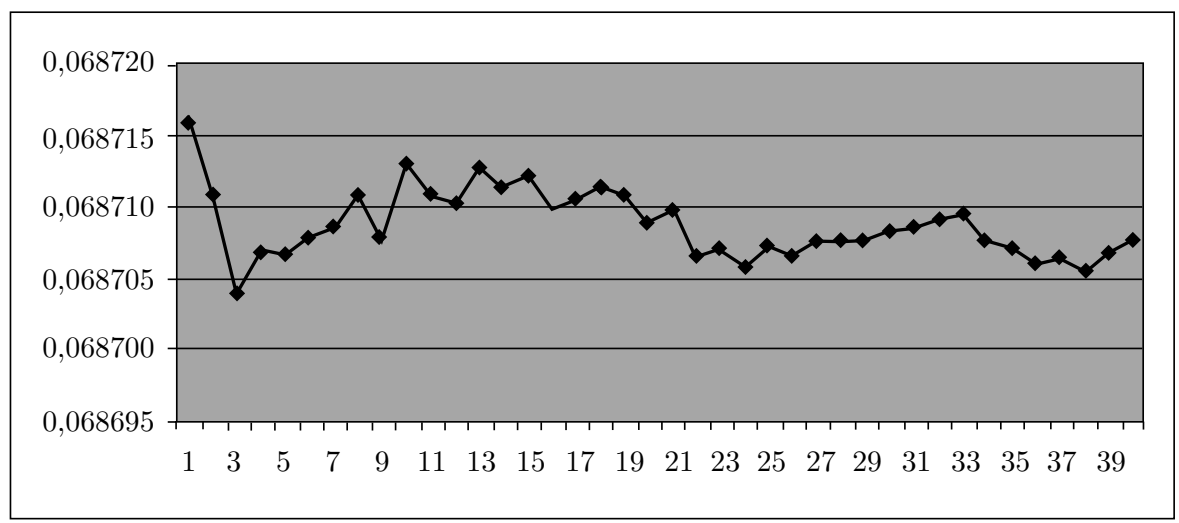

Figure 5. The density $D(7)$ of prime numbers with the least primitive root equal to 7 


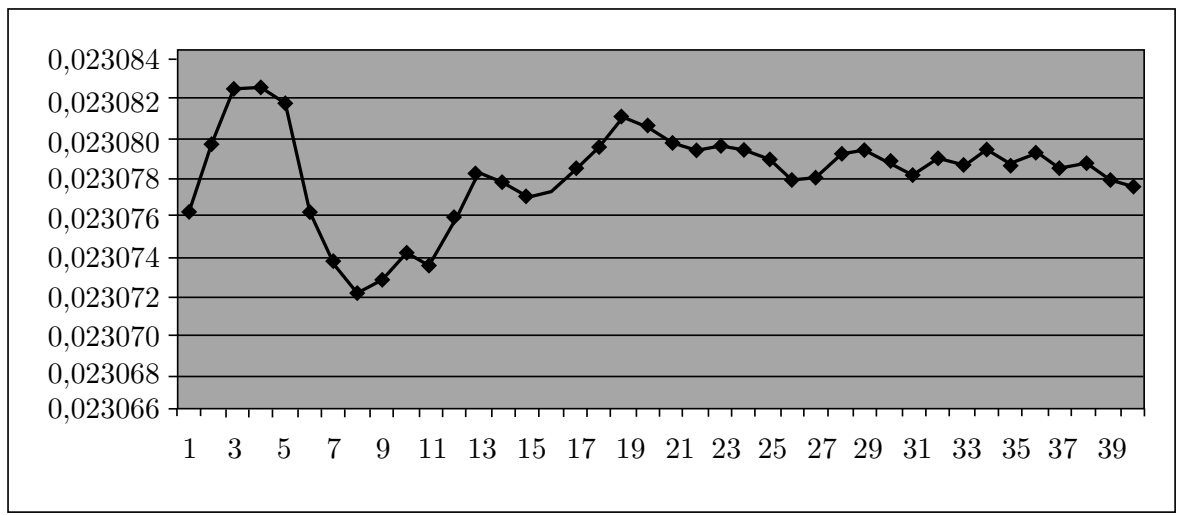

Figure 6 . The density $D(10)$ of prime numbers with the least primitive root equal to 10

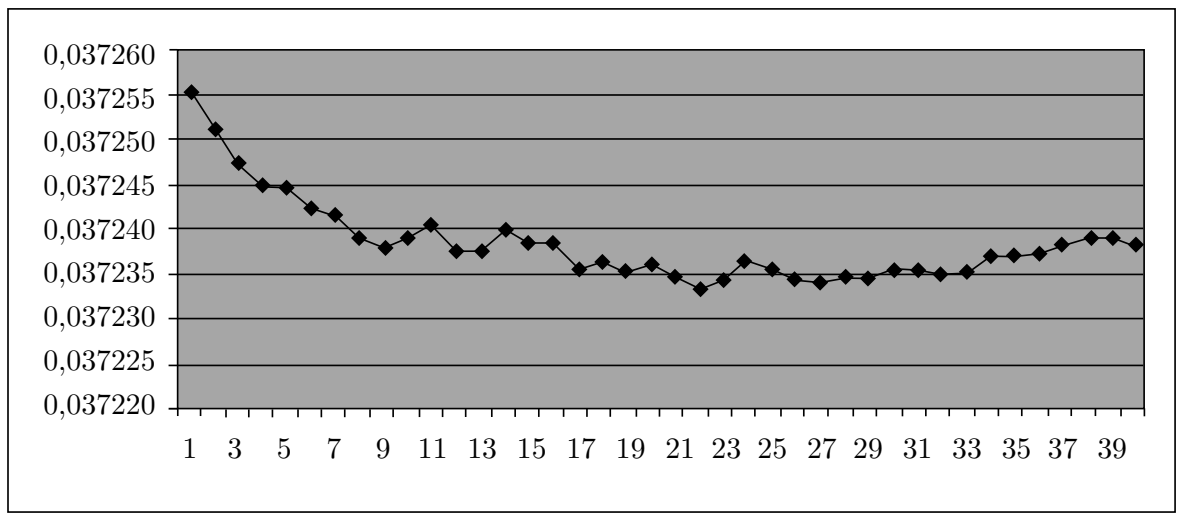

Figure 7 . The density $D(11)$ of prime numbers with the least primitive root equal to 11

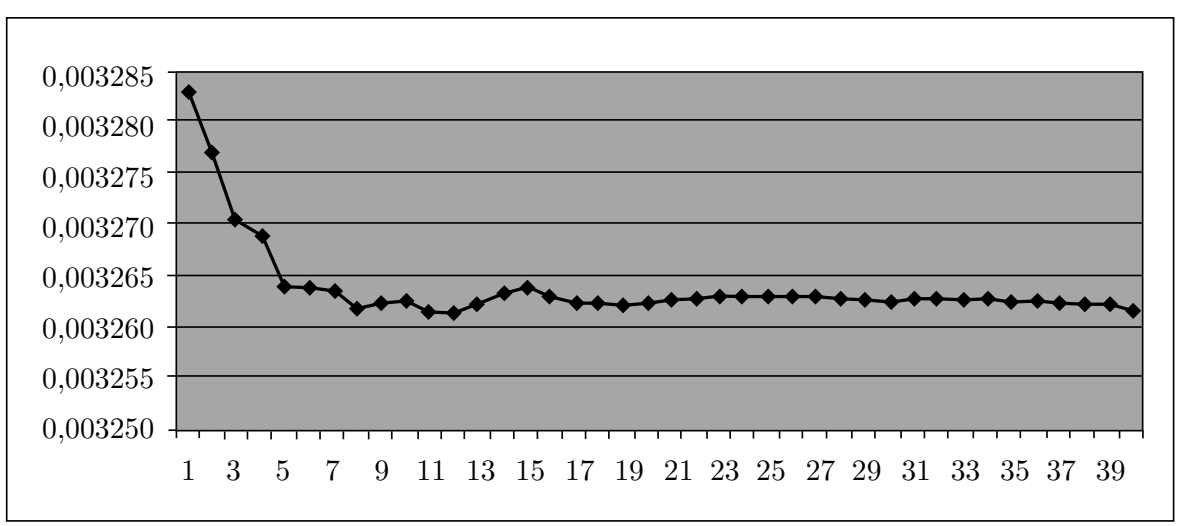

Figure 8 . The density $D(12)$ of prime numbers with the least primitive root equal to 12 


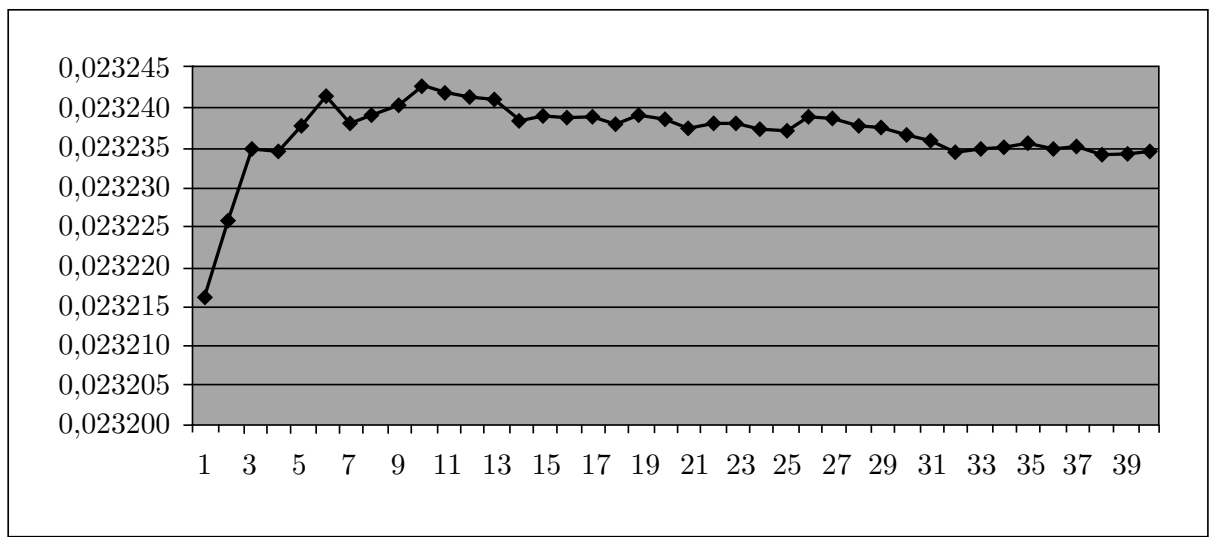

Figure 9 . The density $D(13)$ of prime numbers with the least primitive root equal to 13

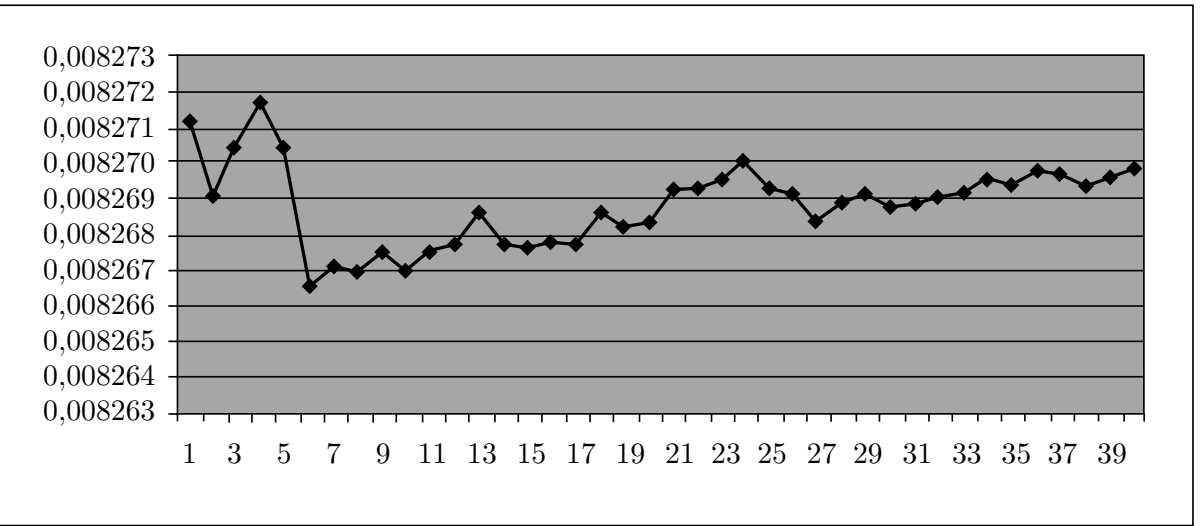

Figure 10. The density $D(14)$ of prime numbers with the least primitive root equal to 14

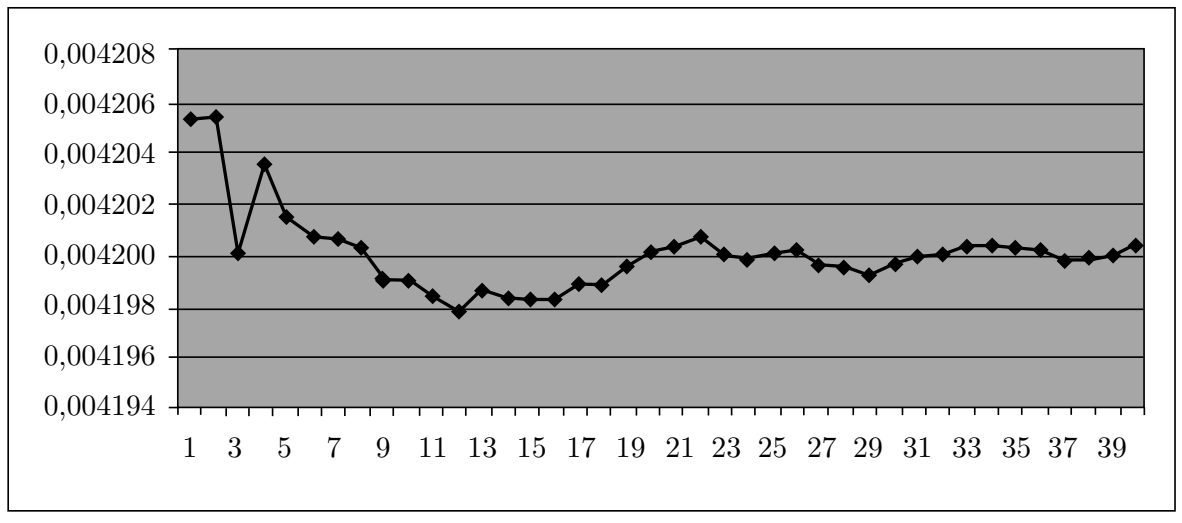

Figure 11 . The density $D(15)$ of prime numbers with the least primitive root equal to 15 


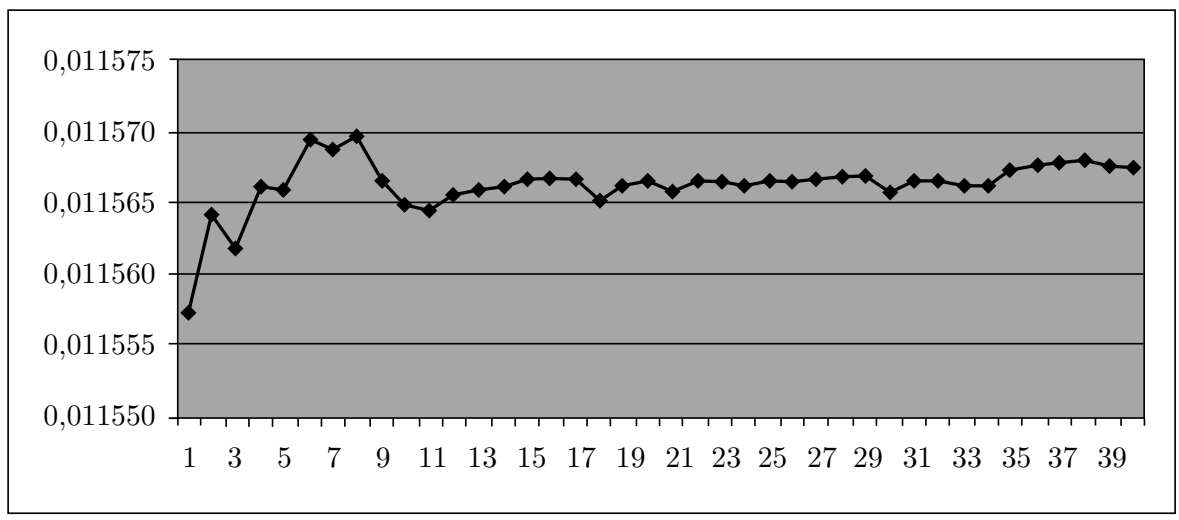

Figure 12. The density $D(17)$ of prime numbers with the least primitive root equal to 17

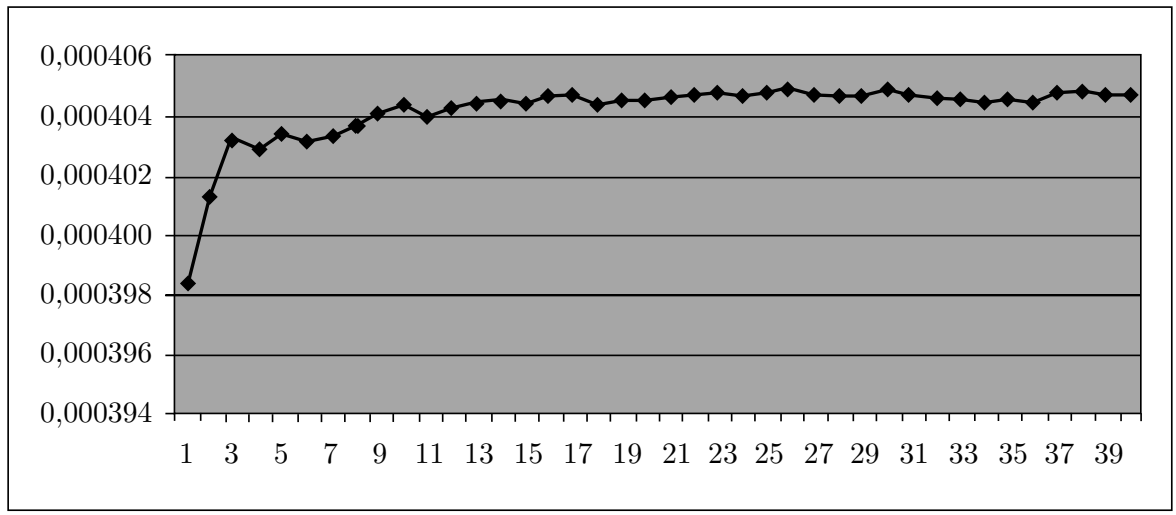

Figure 13. The density $D(18)$ of prime numbers with the least primitive root equal to 18

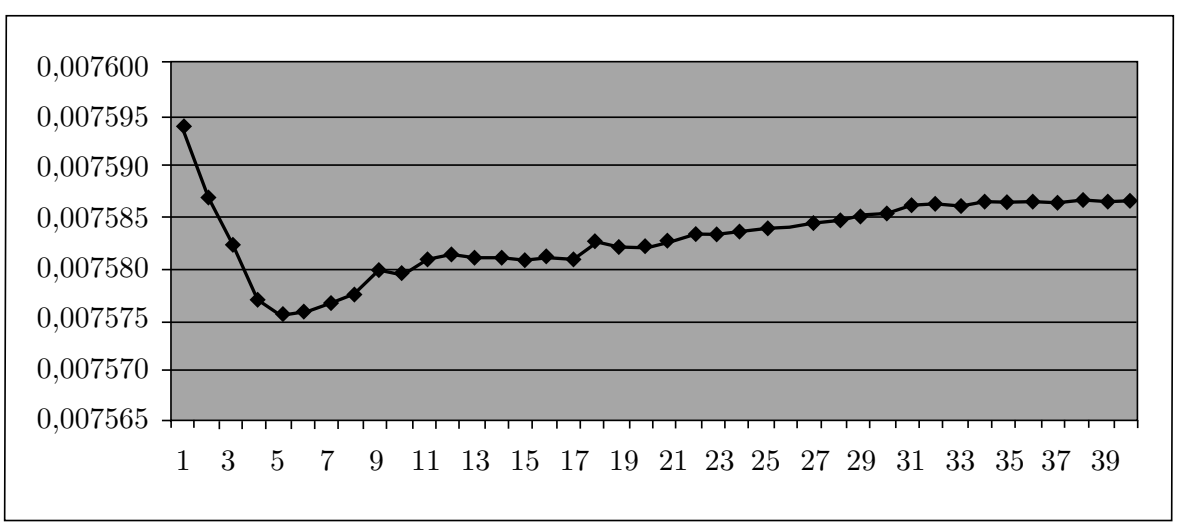

Figure 14. The density $D(19)$ of prime numbers with the least primitive root equal to 19 


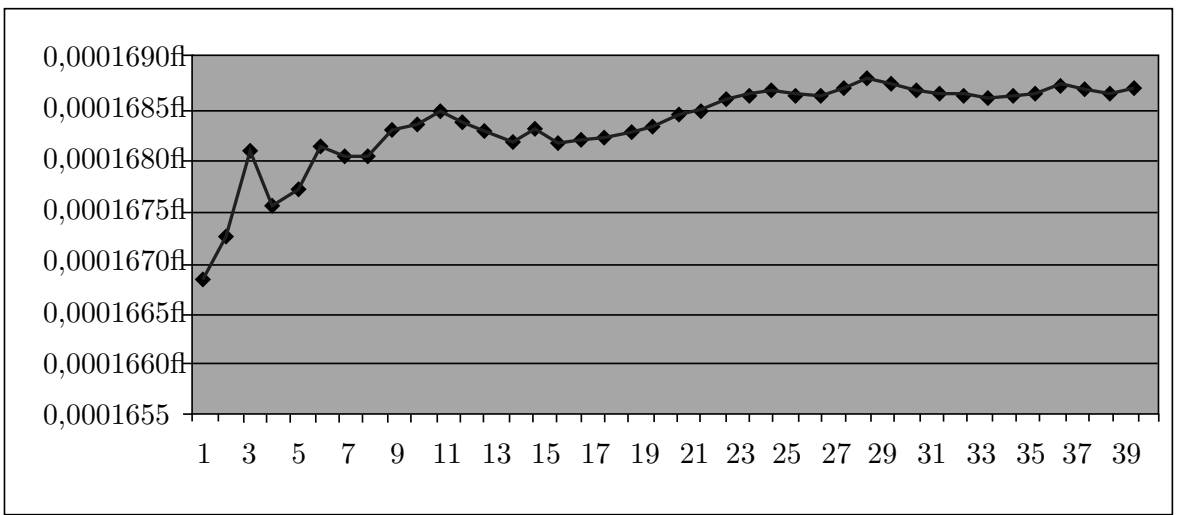

Figure 15 . The density $D(20)$ of prime numbers with the least primitive root equal to 20

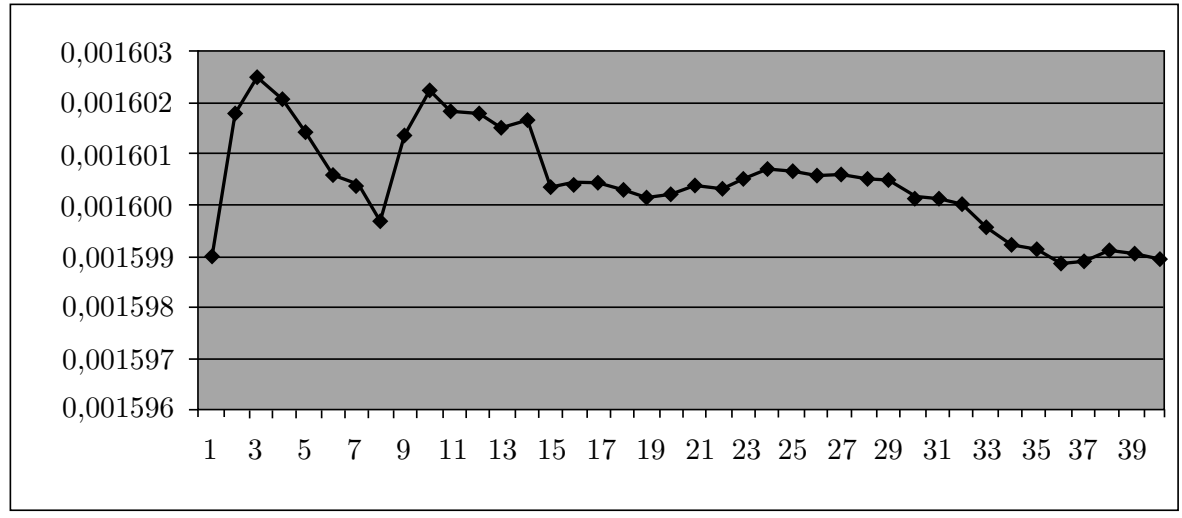

Figure 16 . The density $D(21)$ of prime numbers with the least primitive root equal to 21

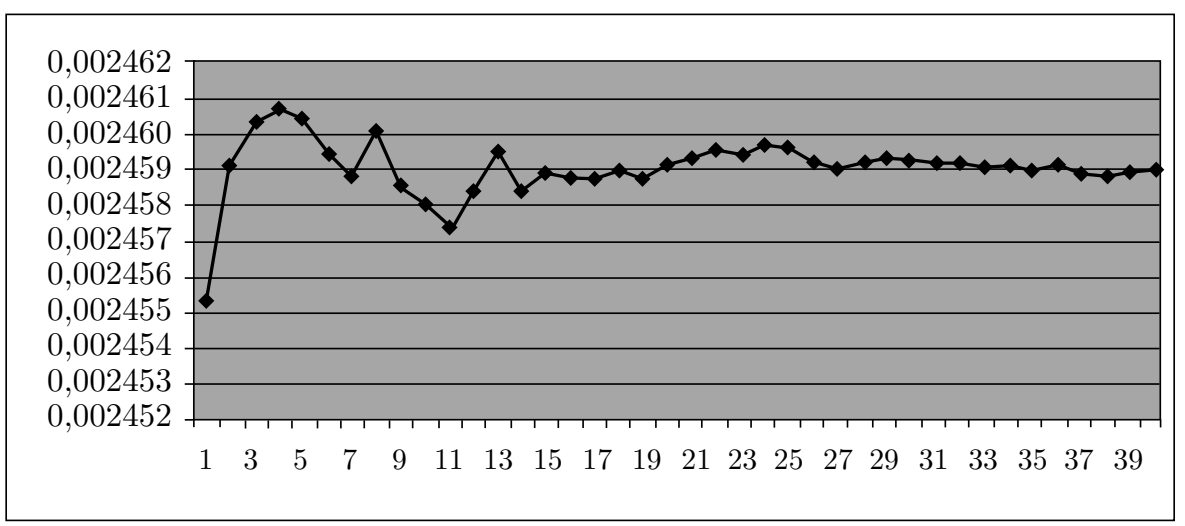

Figure 17 . The density $D(22)$ of prime numbers with the least primitive root equal to 22 


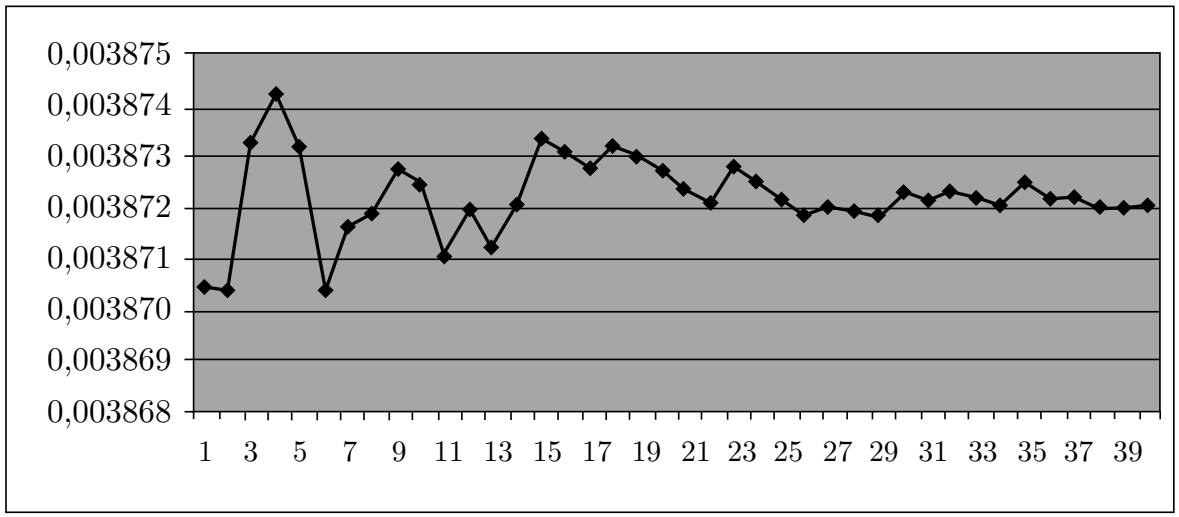

Figure 18. The density $D(23)$ of prime numbers with the least primitive root equal to 23

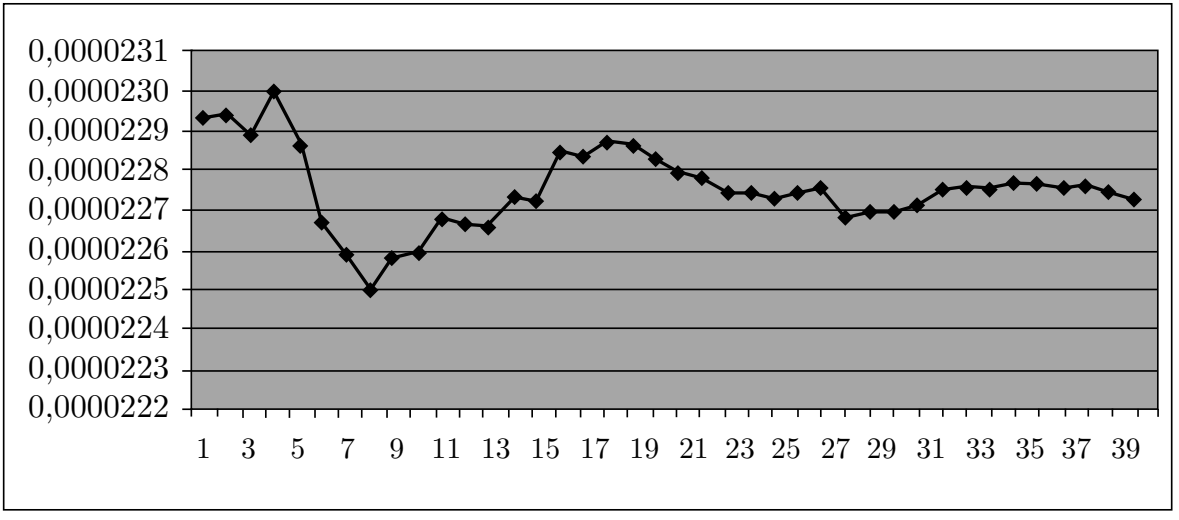

Figure 19. The density $D(24)$ of prime numbers with the least primitive root equal to 24

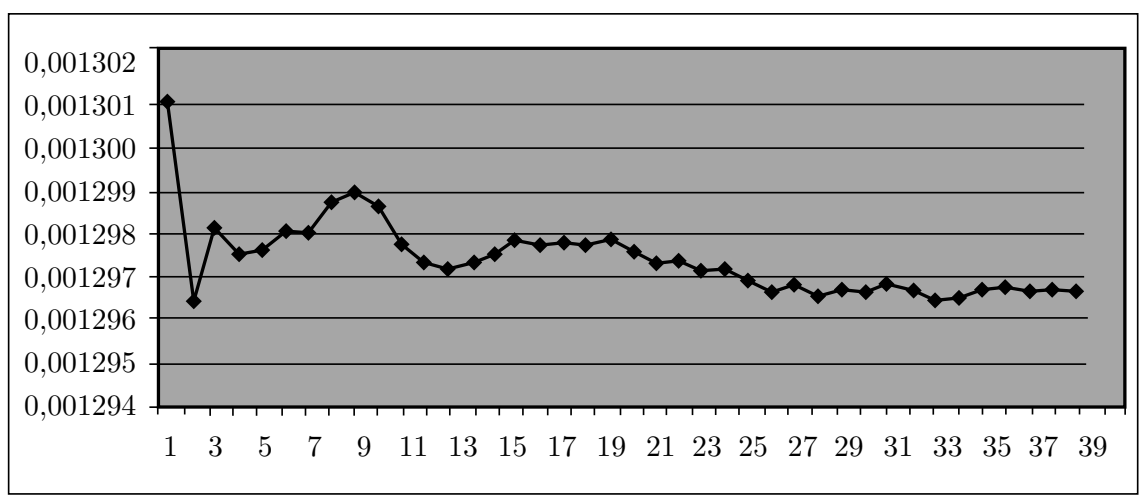

Figure 20. The density $D(26)$ of prime numbers with the least primitive root equal to 26 


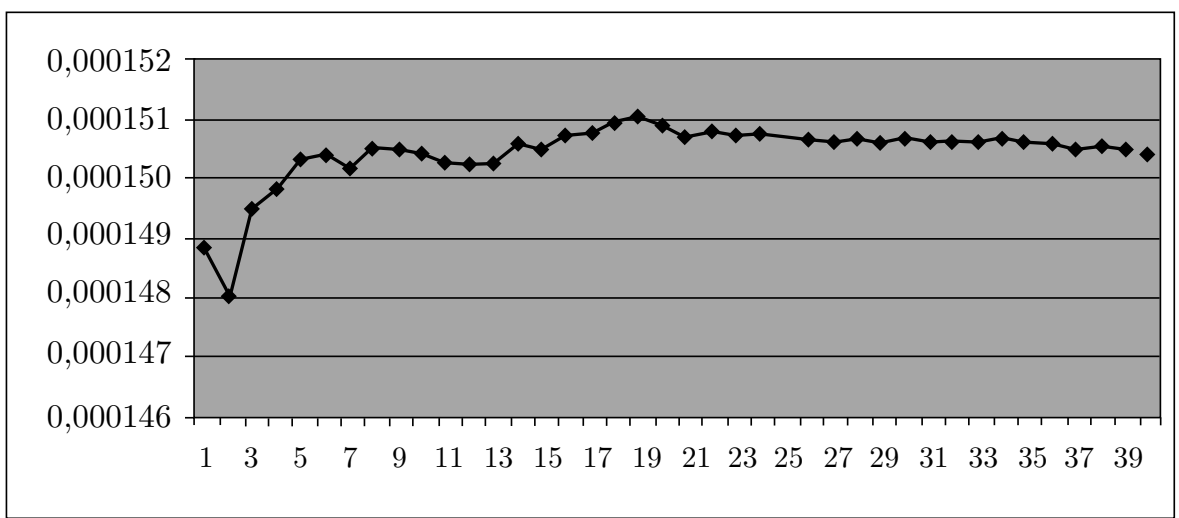

Figure 21. The density $D(28)$ of prime numbers with the least primitive root equal to 28

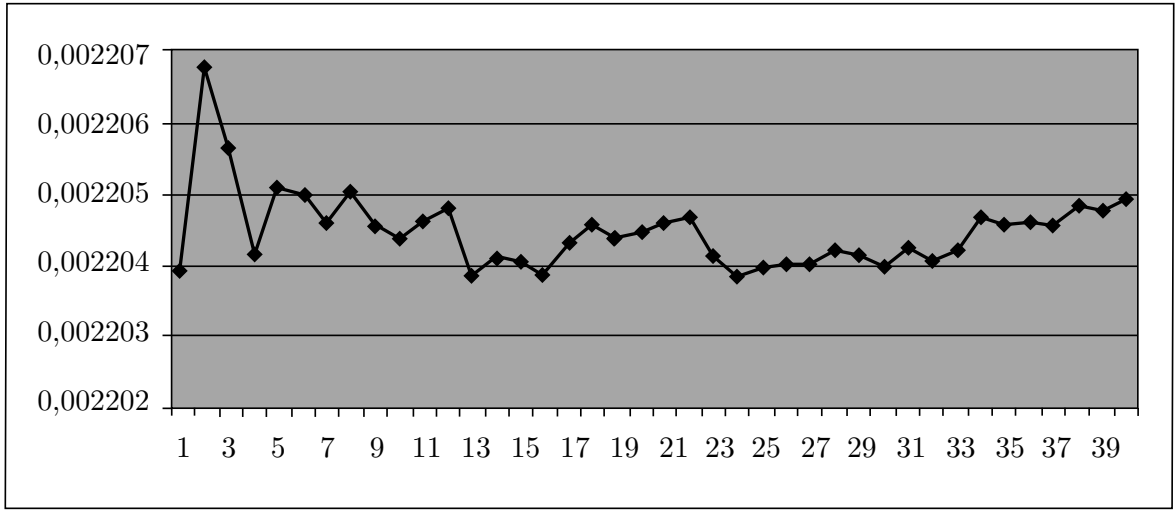

Figure 22. The density $D(29)$ of prime numbers with the least primitive root equal to 29

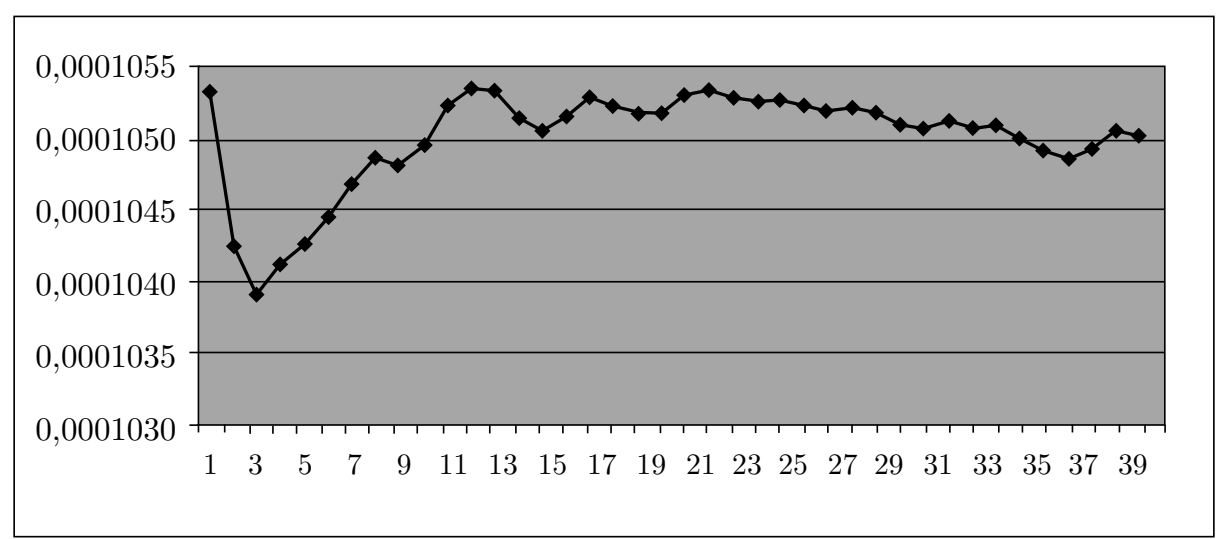

Figure 23. The density $D(30)$ of prime numbers with the least primitive root equal to 30 


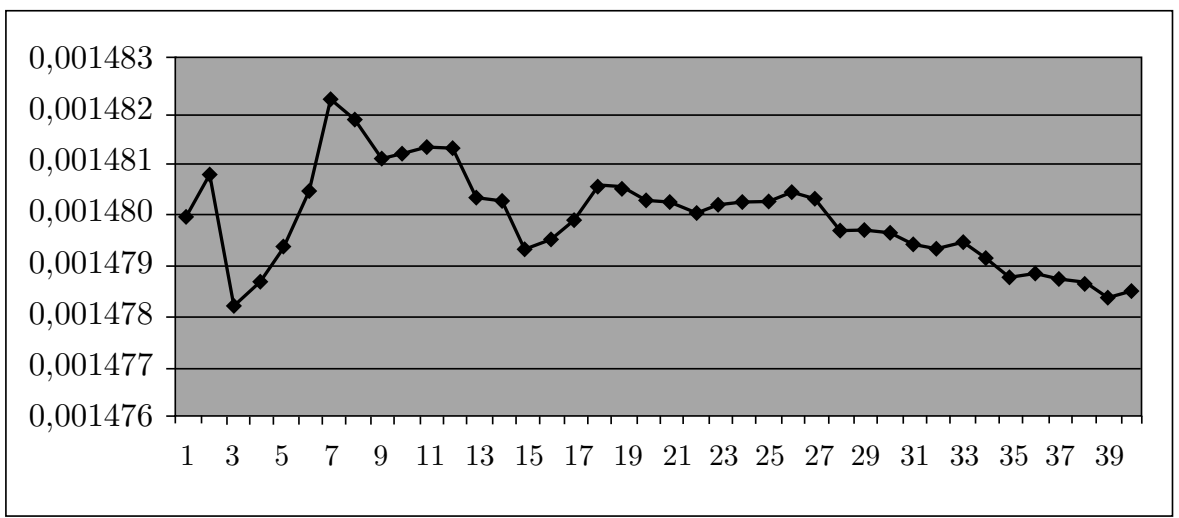

Figure 24 . The density $D(31)$ of prime numbers with the least primitive root equal to 31

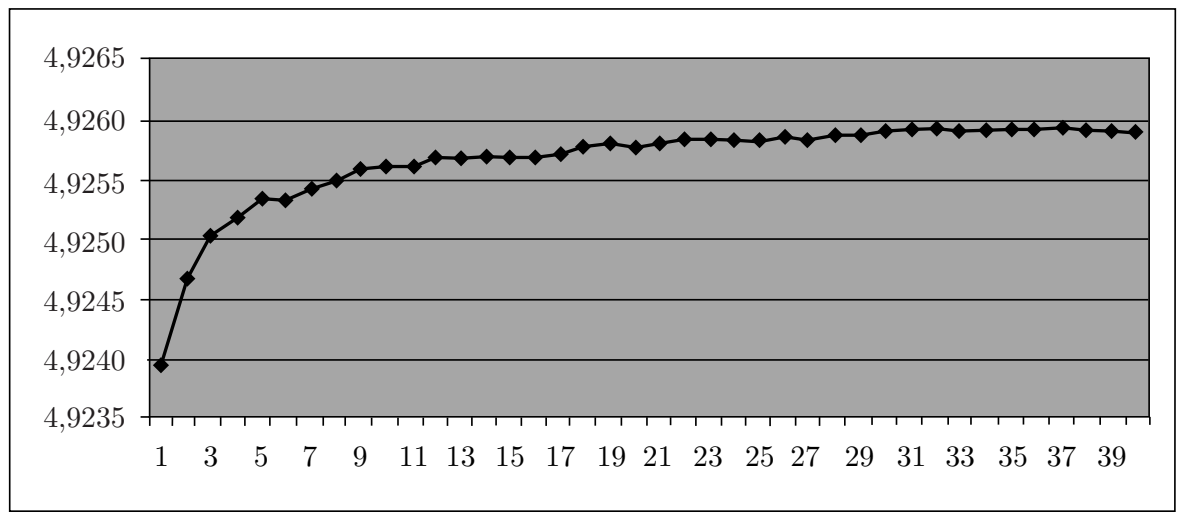

Figure 25. The average value of the least primitive root. The range of computations covers prime numbers less than $4 \cdot 10^{10}$. 
TABLE 1 . The average value of the least primitive root

\begin{tabular}{|r|c|c|c|c|c|}
\hline \multicolumn{1}{r|}{$n$} & $\pi\left(n \cdot 10^{9}\right)$ & $\frac{1}{\pi\left(n \cdot 10^{9}\right)} \sum_{p<n \cdot 10^{9}} g(p)$ & $n$ & $\pi\left(n \cdot 10^{9}\right)$ & $\frac{1}{\pi\left(n \cdot 10^{9}\right)} \sum_{p<n \cdot 10^{9}} g(p)$ \\
\hline 1 & 50847534 & 4.923965712871 & 21 & 924324489 & 4.925797855823 \\
\hline 2 & 98222287 & 4.924663401493 & 22 & 966358351 & 4.925832422385 \\
\hline 3 & 144449537 & 4.925051147795 & 23 & 1008309544 & 4.925843984674 \\
\hline 4 & 189961812 & 4.925188058325 & 24 & 1050186367 & 4.925846754020 \\
\hline 5 & 234954223 & 4.925341307870 & 25 & 1091987405 & 4.925855088048 \\
\hline 6 & 279545368 & 4.925330900136 & 26 & 1133717820 & 4.925866737278 \\
\hline 7 & 323804352 & 4.925440477091 & 27 & 1175385155 & 4.925842229139 \\
\hline 8 & 367783654 & 4.925509101064 & 28 & 1216987937 & 4.925879767368 \\
\hline 9 & 411523195 & 4.925580522868 & 29 & 1258528162 & 4.925900388393 \\
\hline 10 & 455052511 & 4.925611642211 & 30 & 1300005926 & 4.925903390845 \\
\hline 11 & 498388617 & 4.925607016827 & 31 & 1341430624 & 4.925932161364 \\
\hline 12 & 541555851 & 4.925698972831 & 32 & 1382799415 & 4.925928524492 \\
\hline 13 & 584570200 & 4.925693412013 & 33 & 1424115489 & 4.925920066307 \\
\hline 14 & 627440336 & 4.925695173988 & 34 & 1465374659 & 4.925939629611 \\
\hline 15 & 670180516 & 4.925693396312 & 35 & 1506589876 & 4.925939416709 \\
\hline 16 & 712799821 & 4.925704473484 & 36 & 1547756812 & 4.925945180075 \\
\hline 17 & 755305935 & 4.925719780025 & 37 & 1588873108 & 4.925950159639 \\
\hline 18 & 797703398 & 4.925784038089 & 38 & 1629945987 & 4.925942955189 \\
\hline 19 & 840000027 & 4.925795219053 & 39 & 1670972264 & 4.925921554372 \\
\hline 20 & 882206716 & 4.925790644287 & 40 & 1711955433 & 4.925920544687 \\
\hline
\end{tabular}


TABLE 2. The growth rate of the least primitive root modulo a prime number. The range of computations covers all prime numbers less than $4 \cdot 10^{10}$.

\begin{tabular}{|r|r|c|c|c|c|}
\hline$g(p)$ & \multicolumn{1}{|c|}{$p$} & $\frac{g(p)}{\ln (p)}$ & $\frac{g(p)}{\ln ^{2}(p)}$ & $\frac{g(p)}{\ln ^{3}(p)}$ & $\frac{3^{-\gamma} g(p)}{\ln (p)(\ln \ln (p))^{2}}$ \\
\hline 3 & 7 & 1.541695 & 0.792274 & 0.407148 & 1.953083 \\
\hline 5 & 23 & 1.594644 & 0.508578 & 0.162200 & 0.685570 \\
\hline 6 & 41 & 1.615695 & 0.435078 & 0.117159 & 0.527004 \\
\hline 7 & 71 & 1.642159 & 0.385241 & 0.090375 & 0.438590 \\
\hline 19 & 191 & 3.617481 & 0.688745 & 0.131132 & 0.738260 \\
\hline 21 & 409 & 3.492017 & 0.580675 & 0.096558 & 0.609157 \\
\hline 23 & 2161 & 2.995444 & 0.390116 & 0.050807 & 0.404762 \\
\hline 31 & 5881 & 3.571641 & 0.411504 & 0.047411 & 0.429429 \\
\hline 37 & 37761 & 3.510758 & 0.333119 & 0.031608 & 0.355390 \\
\hline 38 & 55441 & 3.478873 & 0.318488 & 0.029157 & 0.341698 \\
\hline 44 & 71761 & 3.935213 & 0.351952 & 0.031477 & 0.379080 \\
\hline 69 & 110881 & 5.939973 & 0.511352 & 0.044020 & 0.554523 \\
\hline 73 & 760321 & 5.390837 & 0.398097 & 0.029398 & 0.445765 \\
\hline 94 & 5109721 & 6.085459 & 0.393966 & 0.025504 & 0.455971 \\
\hline 97 & 17551561 & 5.815119 & 0.348614 & 0.020899 & 0.412241 \\
\hline 101 & 29418841 & 5.873067 & 0.341514 & 0.019858 & 0.407471 \\
\hline 107 & 33358081 & 6.176826 & 0.356571 & 0.020583 & 0.426360 \\
\hline 111 & 45024841 & 6.298685 & 0.357418 & 0.020281 & 0.429585 \\
\hline 113 & 90441961 & 6.168048 & 0.336679 & 0.018377 & 0.409520 \\
\hline 127 & 184254841 & 6.673031 & 0.350624 & 0.018423 & 0.431661 \\
\hline 137 & 324013369 & 6.991117 & 0.356757 & 0.018205 & 0.443395 \\
\hline 151 & 831143041 & 7.352113 & 0.357970 & 0.017429 & 0.451916 \\
\hline 164 & 1685283601 & 7.719390 & 0.363347 & 0.017102 & 0.464042 \\
\hline 179 & 6064561441 & 7.946469 & 0.352773 & 0.015660 & 0.459908 \\
\hline 194 & 7111268641 & 8.551926 & 0.376986 & 0.016618 & 0.492719 \\
\hline 197 & 9470788801 & 8.575852 & 0.373326 & 0.016251 & 0.490148 \\
\hline 227 & 28725635761 & 9.426496 & 0.391448 & 0.016255 & 0.522907 \\
\hline & & & & & \\
\hline
\end{tabular}


TABLE 3. The frequencies of occurrence of prime numbers with a given least primitive root. $D(i)$ denotes the frequency of prime numbers with the least primitive root equal to $i$, calculated with the aid of theoretical considerations.

\begin{tabular}{|c|c|c|}
\hline$i$ & $D(i)$ & $\frac{1}{\pi(a)} \sum_{\begin{array}{c}p<a, g(p)=i \\
\left(a=4 \cdot 10^{10}\right)\end{array}} 1$ \\
\hline 2 & 0.373955 & 0.3739585 \\
\hline 3 & 0.226606 & 0.2266042 \\
\hline 5 & 0.139065 & 0.1390616 \\
\hline 6 & 0.055881 & 0.0558824 \\
\hline 7 & 0.068702 & 0.0687077 \\
\hline 10 & 0.023074 & 0.0230774 \\
\hline 11 & 0.037238 & 0.0372384 \\
\hline 12 & 0.003263 & 0.0032617 \\
\hline 13 & 0.023229 & 0.0232346 \\
\hline 14 & 0.008270 & 0.0082698 \\
\hline 15 & 0.004200 & 0.0042004 \\
\hline 17 & 0.011568 & 0.0115673 \\
\hline 18 & 0.000404 & 0.0004047 \\
\hline 19 & 0.007586 & 0.0075864 \\
\hline 20 & 0.000168 & 0.0001687 \\
\hline 21 & 0.001600 & 0.0015989 \\
\hline 22 & 0.002459 & 0.0024589 \\
\hline 23 & 0.003873 & 0.0038720 \\
\hline 24 & 0.000022 & 0.0000227 \\
\hline 26 & 0.001297 & 0.0012966 \\
\hline 28 & 0.000150 & 0.0001503 \\
\hline 29 & 0.002203 & 0.0022049 \\
\hline 30 & 0.000104 & 0.0001050 \\
\hline 31 & 0.001479 & 0.0014784 \\
\hline
\end{tabular}




\section{Acknowledgments}

The referee informed us that in the Ph.D. thesis of Bob Buttsworth, University of Queensland 1983 [2], the formula for $D(i)$ is transformed using finite difference methods, into a form which allows one to prove that $D(i)$ is positive if $i$ is not a perfect power, for which we are grateful.

The first author was technically supported during computations by the grant of Polish Committee for Scientific Research Nr. 8 T11 D 01112.

\section{REFERENCES}

1. E. Bach, Comments on search procedures for primitive roots, Math. Comp. 66 (1997), 17191727. MR 98a:11187

2. R. N. Buttsworth, A general theory of inclusion-exclusion with application to the least primitive root problem, and other density question, Ph.D. Thesis, University of Queensland, Queensland, 1983.

3. P.D.T.A. Elliott, L. Murata, On the average of the least primitive root modulo $p$, J. London Math. Soc. (2) 56 (1997), 435-454. MR 98m:11094

4. K. R. Matthews, A generalisation of Artin's conjecture for primitive roots, Acta Arith. 29 (1976), 113-146. MR 53:313

Warsaw University of Technology, Institute of Telecommunications, Division of Telecommunications Fundamental, ul. Nowowiejska 15/19, 00-665 WarsaW, Poland

E-mail address: anpa@tele.pw.edu.pl

Institute of Mathematics, Polish Academy of Sciences, ul. Śniadeckich 8, 00-950 WarSAW, POLAND

E-mail address: schinzel@impan.gov.pl 